\title{
Protocol S1:Mechanochemical coupling in the myosin motor domain. I. Insights from equilibrium active-site simulations
}

\section{A Sidechain distribution of P-loop, Switch I and Switch II}

To investigate the effect of ATP hydrolysis on the local flexibility of active-site residues, the sidechain distributions (Fig. S1-S3) are analyzed for both the ATP and ADP.P $P_{i}$ state with the $1 \mathrm{VOM}$ simulations (closed active-site). In addition, the same quantities are also

analyzed for the 1VOM-ATP simulation with a larger inner region ( $32 \AA$ instead of $20 \AA$ ) to investigate whether the size of the GSBP inner region has a major impact on the active-site flexibility. As seen in Fig. S1-S3, little difference is found between simulations of different inner region size, except for a few residues and the effect is small in all cases. As to the effect of ATP hydrolysis, the only residue that has been substantially affected is Ser 237, which has a much broader distribution in the ADP. $\mathrm{P}_{i}$ state, suggesting that the interaction with $\mathrm{Mg}^{2+}$ is substantially reduced.

\section{B Convergence of the active-site open/close PMFs}

As shown in Fig. S4, the calculated PMFs with nearly 4-5 times more simulation data are essentially identical with the original PMFs. This ensures that the computed PMFs are converged to a satisfactory degree.

\section{Figure Captions}

Fig.S1-S3 Sidechain distributions in the P-loop (Fig. S1), Switch I (Fig. S2) and Switch II (Fig. S3) residues from different simulations. 
Fig. S4 Potentials of mean force for the open/close of the active site in the (a) 1FMW-ATP and (b) 1VOM-ATP simulations with different length of trajectories. 
1VOM:ATP $(20 \AA)$

1VOM:ADP.Pi (20 Å)

1VOM:ATP (32 Å)
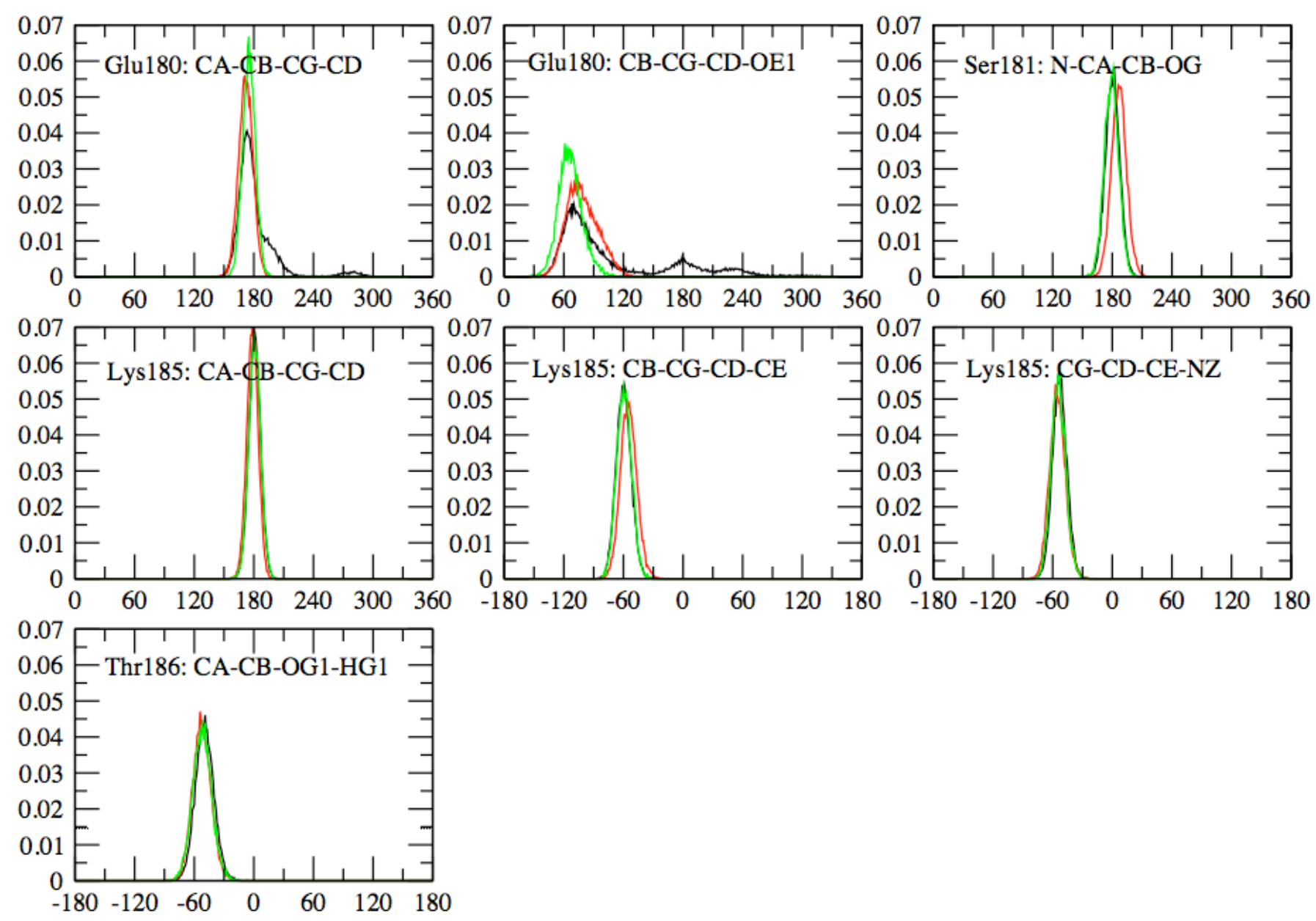

Fig. S1 
1VOM:ATP $(20 \AA)$

1VOM:ADP.Pi (20 Å)

1VOM:ATP (32 ̊)
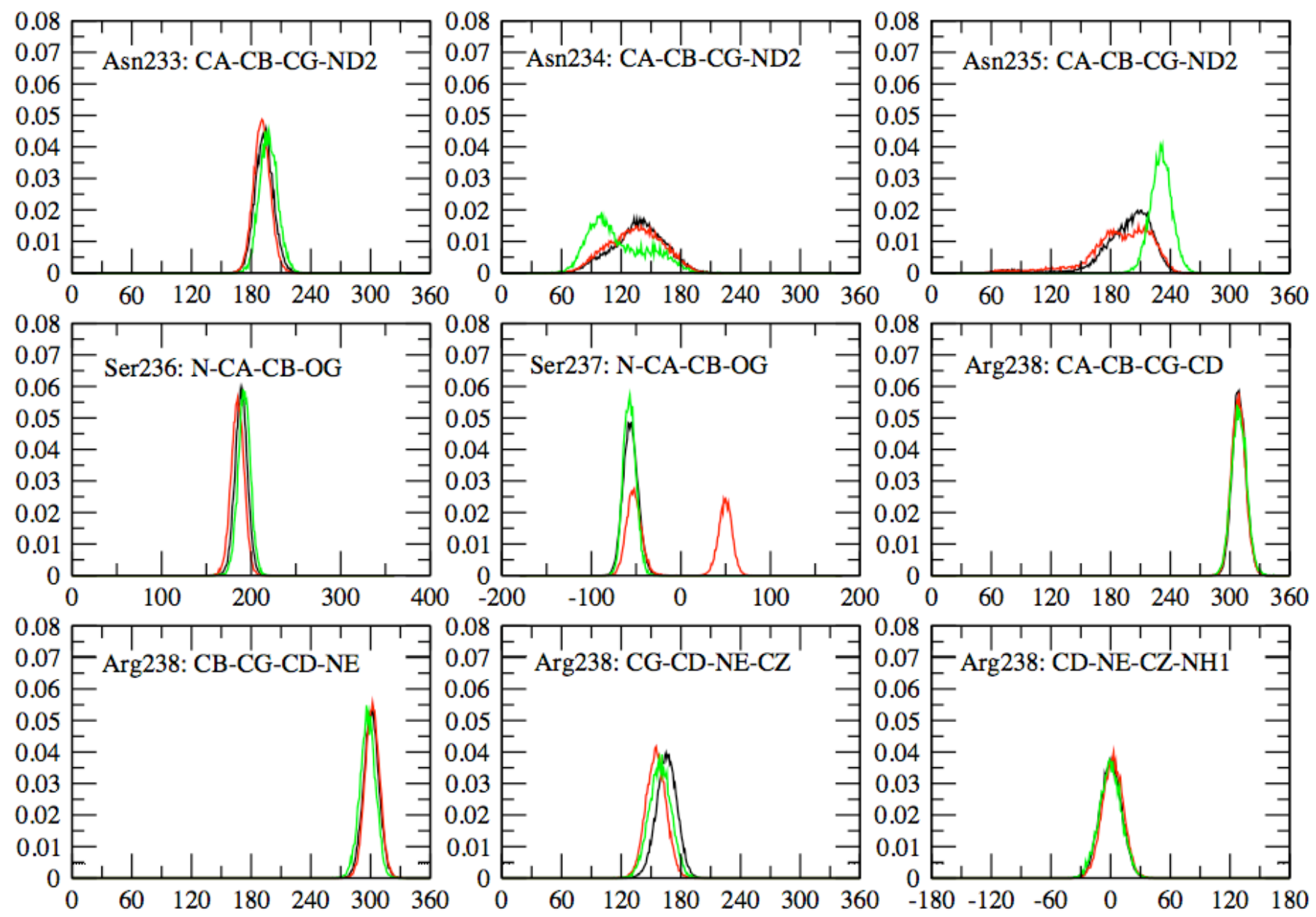

Fig. S2 
1VOM:ATP (20 ̊)

1VOM:ADP.Pi (20 Å)

1VOM:ATP (32 ̊)
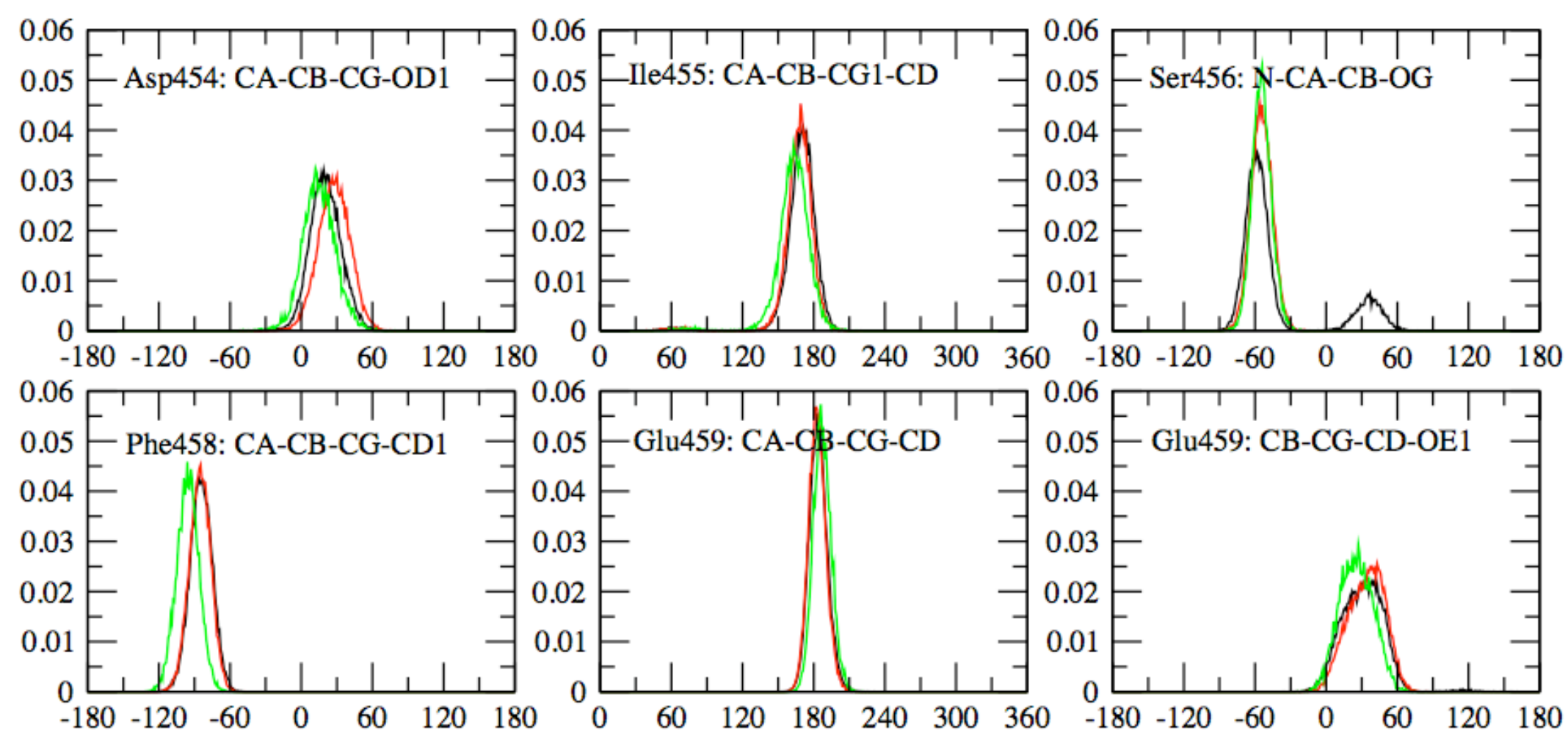

Fig. S3 

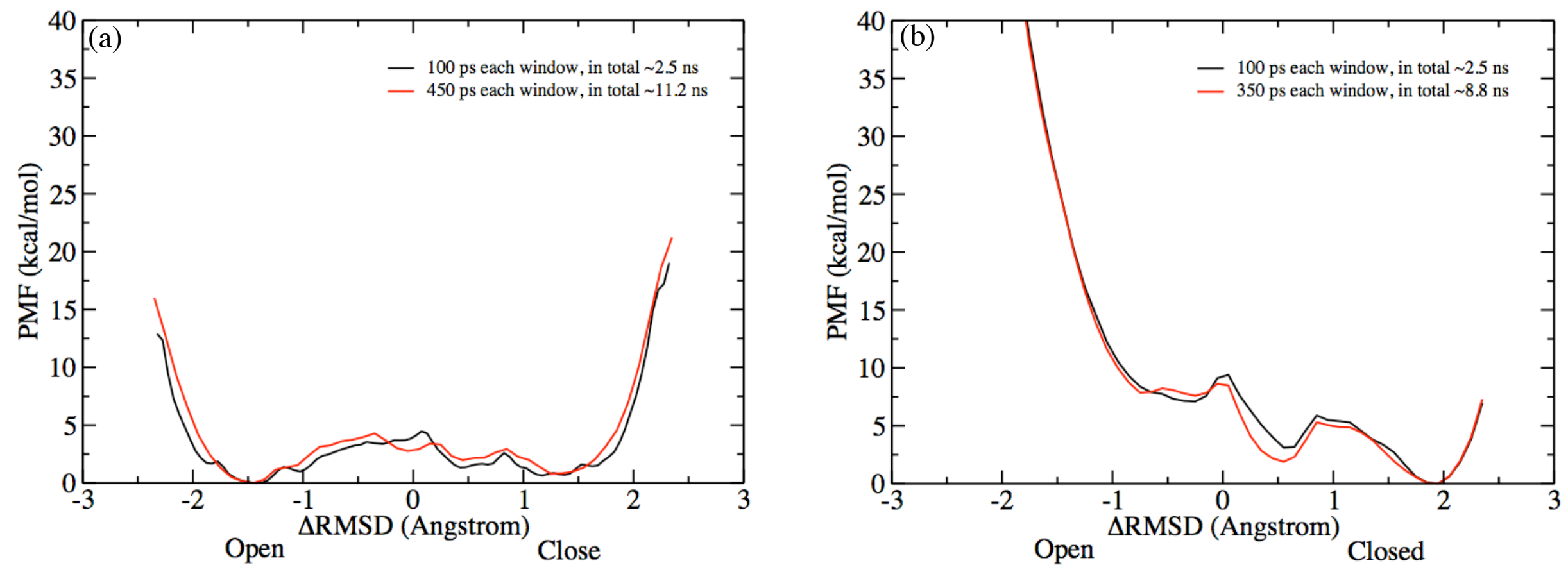

Fig. S4 